\title{
The Surgeon's Role in the Quality of Oncologic Care
}

Quality should be the right treatment for the right patient at the right time. In response to widely varying patient outcomes, national cancer organizations are emphasizing improvement in the quality of cancer care. The National Quality Forum, National Comprehensive Cancer Network, American Society of Clinical Oncology, and American College of Surgeons' Commission on Cancer, for example, have all defined quality measures and performed pilot projects to show how to measure and improve quality. Additionally, recent pay-for-performance initiatives have brought even more attention to quality improvement. Because cancer care is complex and multidisciplinary, surgeons must play an integral role in quality improvement.

Providing good quality surgical care is essential and involves several considerations. This commentary discusses 6 of them.

\section{Recognize the population}

People older than 65 years comprise the fastest growing population in the United States. The U.S. Census Bureau estimates that they will represent 13\% of the population by 2010 and more than $50 \%$ by 2020. In 1999, they accounted for $40 \%$ of hospital discharges and $48 \%$ of inpatient care days. ${ }^{1}$ Because the risk for most cancers increases with age, this group represents a large cohort of patients who will need oncologic care. Moreover, this group of patients has unique issues, such as the need for evaluating their decision-making capacity, drafting of a living will or durable power of attorney, evaluation of functional status, need for assistance with activities of daily living (ADLs) postoperatively, and the management of polypharmacy. These issues, along with complex comorbidities, underscore the need for a multidisciplinary team approach to cancer care in the elderly.

\section{Evaluate and Manage the Comorbidities}

The number and severity of comorbidities increase as patients age. ${ }^{2}$ Therefore, to provide high-quality surgical care, evaluating and optimizing comorbidities is important. Although surgeon volume is traditionally associated with outcome, the acuity of surgery and patient comorbidities are possibly more predictive. ${ }^{3}$ Patients undergoing emergent surgery have been shown to be nearly 4 times more likely to die during the hospital course than those undergoing elective surgery. Similarly, patients with cardiac, pulmonary, and hepatic comorbidities had a substantial increase in mortality. ${ }^{3}$ These factors highlight the importance of a thorough preoperative evaluation.

\section{Perform Appropriate Processes}

"Processes of care" are what providers do for their patients. These efforts range from obtaining an appropriate history and performing a physical examination to appropriately offering neoadjuvant therapy and to taking necessary actions in the operating room. Quality-of-care studies have used process measures to

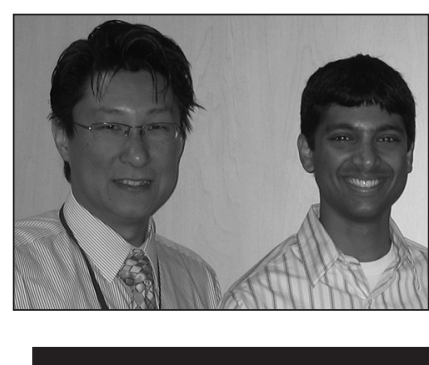

Clifford Ko, MD, MS, MSHS and Janak Parikh, MD

Clifford Ko, MD, MSHS (left), is an Associate Professor and Vice Chair in the Department of Surgery at UCLA, Chief of Colorectal Surgery at the West Los Angeles Veteran's Affairs (WLAVA) and Medical Director of the National Cancer Data Base.

Janak Parikh, MD (right), is a fellow in the UCLA Center for Surgical Outcomes and Quality.

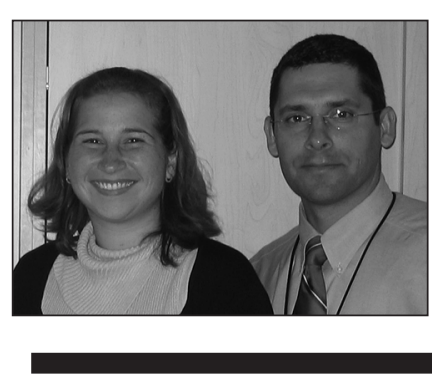

Irina Yermilov, MD, MPH and James Tomlinson, MD Irina Yermilov, MD, MPH (left), is a fellow in the UCLA Center for Surgical Outcomes and Quality. James Tomlinson, MD (right), is an Assistant Professor of Surgery and Director of the Cancer Program at the West Los Angeles Veteran's Affairs.

The ideas and viewpoints expressed in this commentary are those of the authors and do not necessarily represent any policy, position, or program of the NCCN. 
Ko, et al.

evaluate quality. For example, the National Initiative for Cancer Care Quality project found that patients with breast and colorectal cancer received $86 \%$ and $78 \%$ of recommended care, respectively. ${ }^{4}$ For surgeons caring for cancer patients, numerous important processes of care occur in the preoperative, intraoperative, and postoperative periods.

\section{Preoperative Planning}

In addition to evaluating and optimizing comorbid conditions preoperatively, physicians must consider other issues. McGory et al. ${ }^{5}$ recently published quality indicators for elderly patients undergoing abdominal surgery. According to these evidence-based, expert-panel-verified quality indicators, the patient's nutritional status, cognitive function, ambulatory ability, functional status, and medication list should be evaluated preoperatively. During the preoperative assessment, the surgeon must also select the most suitable operation for the patient. This requires a frank discussion with the patient about the disease, the role of surgery (cure vs. palliation), potential complications, overall prognosis, and postoperative functional status. Additionally, an advance directive or living will indicating the patient's preferences should be placed in the chart. ${ }^{5}$

\section{Intraoperative Management}

In the operating room, surgeons must perform the appropriate processes specific to the surgery. For example, key steps in performing an appropriate resection of a low rectal cancer include adequate exposure, identification of the hypogastric nerves and left ureter, appropriate mobilization of the left colon, and a total mesorectal excision. If a sphincter-sparing operation is performed, several processes of care are also involved with performing the anastomosis. Aside from processes for specific operations, generic processes such as maintaining of intraoperative normothermia and proper positioning on the operating room table must also be done to avoid compressive neuropathy. ${ }^{5}$

\section{Postoperative Period}

Processes in the postoperative period include prevention of iatrogenic complications and discharge planning. Important additional quality measures in the postoperative period include close monitoring of fluid status, elevating the head of the bed, performing incentive spirometry, and preventing infection with prophylactic antibiotics. Experts also suggest reevaluating certain preoperative measures, includ- ing the patient's home environment, cognition, functional status, ambulatory ability, and nutrition, before discharge. ${ }^{5}$ This is critical because Lawrence et al. ${ }^{6}$ showed that 6 months after major abdominal surgery, a significant incidence of disability remained in elderly patients in regard to ADLs, ambulation, cognition, and physical strength and conditioning.

\section{Measure and Know the Outcomes}

Traditional outcome measures examine patient morbidity and mortality after treatment. However, patient-reported items, such as quality of life, functional capacity, ability to perform ADLs, and satisfaction, may be equally important. ${ }^{7}$ Recording outcomes, regardless of the measure, allows surgeons to recognize areas for improvement and to design and implement interventions to improve quality. ${ }^{8}$

\section{Work as a Team}

Given the increasing complexity of multidisciplinary cancer care, efficient delivery of stage-appropriate treatment requires coordination among multidisciplinary team members. Teamwork in cancer care involves each team member understanding the full spectrum of care for the cancers treated. This is important because the patient may initially present to any member of the team. Understanding the totality of care allows each member to direct the patient to the appropriate next step in the treatment algorithm. For example, if a patient with stage 3 rectal cancer initially presents to a surgeon, the surgeon should know that the patient should be referred for neoadjuvant therapy before surgery. Stage-appropriate care can be assured through a multidisciplinary conference that would also facilitate communication among team members, which is critical to providing good quality care.

\section{Know Your Limitations}

An important aspect of quality improvement is having the most expert and experienced staff available to care for patients with specific diseases. That specialist providers who treat a high volume of patients with a particular condition consistently have better outcomes than generalists is well established..$^{9-11}$ The reasons for this are likely related to a more thorough and current knowledge of the literature and more experience treating 
the illness and its potential complications. This even has implications within subspecialties, where one surgical oncologist may specialize in the treatment of soft tissue sarcomas, whereas another focuses on melanoma. In an era when quality of care is at the forefront, surgeons probably should not promote a "jack of all trades, master of none" philosophy. Instead, surgeons should aspire to be a master of some. Currently, the definition of some-how much experience one needs to be deemed a specialist-is unclear. However, as more data on quality are collected and evaluated and as this information becomes increasingly more transparent, the resolution of this debate will become clearer.

\section{References}

1. Liu JH, Etzioni DA, O'Connell JB, et al. The increasing workload of general surgery. Arch Surg 2004;139:423-428.

2. Guralnik JM, LaCroix AZ, Everett DF, Kovar MG. Aging in the eighties: the prevalence of comorbidity and its association with disability. Advance Data 1989;170:1-8.
3. Ko CY, Chang JT, Chaudhry S, Kominski G. Are high-volume surgeons and hospitals the most important predictors of in-hospital outcome for colon cancer resection? Surgery 2002;132:268-273.

4. Malin JL, Schneider EC, Epstein AM, et al. Results of the National Initiative for Cancer Care Quality: how can we improve the quality of cancer care in the United States? J Clin Oncol 2006;24:626-634.

5. McGory ML, Shekelle PG, Rubenstein LZ, et al. Developing quality indicators for elderly patients undergoing abdominal operations. J Am Coll Surg 2005;201:870-883.

6. Lawrence VA, Hazuda HP, Cornell JE, et al. Functional independence after major abdominal surgery in the elderly. J Am Coll Surg 2004;199:762-772.

7. Avery KN, Metcalfe C, Nicklin J, et al. Satisfaction with care: an independent outcome measure in surgical oncology. Ann Surg Oncol 2006;13:817-822.

8. Khuri SF, Daley J, Henderson WG. The comparative assessment and improvement of quality of surgical care in the Department of Veterans Affairs. Arch Surg 2002;137:20-27.

9. Schreiber TL, Elkhatib A, Grines CL, O'Neill WW. Cardiologist versus internist management of patients with unstable angina: treatment patterns and outcomes. J Am Coll Cardiol 1995;26:577-582.

10. Levetan CS, Passaro MD, Jablonski KA, Ratner RE. Effect of physician specialty on outcomes in diabetic ketoacidosis. Diabetes Care 1999;22:1790-1795.

11. Nash IS, Corrato RR, Dlutowski MJ, et al. Generalist versus specialist care for acute myocardial infarction. Am J Cardiol 1999;83:650-654. 